\title{
A comparison of cleavage-stage embryo transfer and blastocyst transfers in patients with low anti-Mullerian hormone levels
}

\author{
๑Nur Dokuzeylül Güngörr ${ }^{1}$, \Tuğba Gürbüz ${ }^{2}$, ๑Arzu Yurci ${ }^{3}$ \\ ${ }^{1}$ Bahçeşehir University, Göztepe Medikal Park Hospital Complex, Gynecology and IVF Clinic, İstanbul, Turkey \\ ${ }^{2}$ Medistate Hospital, Gynecology and Obstetric Clinic, İstanbul, Turkey \\ ${ }^{3}$ Memorial Hospital, IVF Clinic, Kayseri, Turkey
}

Cite this article as: Dokuzeylül Güngör N, Gürbüz T, Yurci A. A comparison of cleavage-stage embryo transfer and blastocyst transfers in patients with low anti-Mullerian hormone levels. Anatolian Curr Med J 2021; 3(4); 264-268.

\begin{abstract}
Aim: Decreased ovarian reserve is a common problem that many women struggle with and seek help by assisted reproductive techniques (ART). Embryo transfer (ET) is an important step in ART that can be performed on cleavage-stage (day 2 or 3 ) or blastocyst stage (day 5 or 6 ). This study aims to investigate the effect of ET day on pregnancy results in patients with low levels of anti-Mullerian hormone (AMH).

Material and Method: This retrospective study enrolled 239 women with low AMH levels referred to the Bahçeşehir University, Göztepe Medikal Park Hospital In-vitro Fertilization (IVF) Clinic from May 2015 to April 2020. The patients were divided into two groups: blastocyst transfer group and cleavage-stage transfer group. Among the participants, blastocyst transfer was performed in 30 women and cleavage-stage ET on 209 women, respectively.

Results: Among 239 participants, 30 (12.6\%) were in the blastocyst group, and 209 (87.4\%) were in the cleavage-stage group. There was no significant difference in the total number of retrieved oocytes between the two groups $(\mathrm{p}=0.1)$. The number of mature oocytes (MII) was significantly different between the two groups $(p=0.006)$. Examining pregnancy results between the two groups showed no significant difference between positive and negative pregnancy tests $(\mathrm{p}=0.4)$. Even though a higher live birth rate for the blastocyst transfer group was observed with 15(50\%), no significant difference was found between the number of live births and miscarriages in the two groups $(\mathrm{p}=0.1)$.
\end{abstract}

Conclusion: The transfer day does not affect the pregnancy results in women with low AMH levels.

Keywords: Anti-Mullerian hormone, assisted reproductive technique, blastocyte, cleavage, embryo transfer

\section{INTRODUCTION}

Infertility is a problem that one in six couples struggles with and may seek help with assisted reproductive technique (ART). About $30-40 \%$ of the causes of infertility in women are related to ovarian disorders, and the rest are related to uterine disorders, immune factors, and systemic diseases (1). The cause of infertility has always been discussed in ART treatments, and patients with various causes such as endometriosis, male factor, tubular causes, and polycystic ovary syndrome (PCOS) are treated, and the effect of hormonal disorders has always been discussed $(2,3)$.

Anti-Mullerian hormone (AMH) is a bi-chain glycoprotein from the family of cell growth and differentiation factors. AMH levels are associated with the number of primary follicles and have a higher specificity than the levels of luteinizing hormone (LH), follicle- stimulating hormone (FSH), and estradiol on the third day of the menstrual cycle (2). Unlike FSH, which must be measured on the second or third day of menstruation, $\mathrm{AMH}$ can be measured on any day of the cycle. AMH level reflects ovarian function, and it decreases with age. Also, the AMH level is low in women who do not respond well to ovarian stimulation drugs in in-vitro fertilization (IVF) programs (2-4).

Embryo Transfer (ET) from the laboratory to the uterus is an important step in ART. Traditionally, ET is done on day 2-3 or cleavage-stage, but with the advancement of IVF and embryo culture in recent decades, blastocyst transfer was preferred (5). It is said that blastocyst transfer may be physiologically more appropriate because it is closer to the time of natural implantation $(6,7)$. In the recent decade, blastocyst transfer in ART cycles has 
increased significantly (8). Simultaneously with this increase in the use of blastocysts transfer, other advances have been made in the fields of assisted reproduction, such as sequential culture media, time-lapse systems, and vitrification programs to replace freezing techniques that enable pre-implantation genetic screening. These advances have led to the re-discussion of the cleavagestage transfer as an effective method instead of the blastocyst transfer method (13-15). Therefore, this study aimed to investigate the effectiveness of transfer day on pregnancy results in patients with low AMH levels.

\section{MATERIAL AND METHOD}

Ethical approved this study by Research and Project Development Ethics Committee of Beykoz University (Date: 21.12.2020, Decision No: 2020/04). All procedures conducted in our study conformed to the national or institutional research committee's ethical standards and the 1964 Helsinki Declaration and its later amendments.

This retrospective study included 239 infertile women referred to the Bahçeşehir University Göztepe Medical Park Hospital IVF Clinic from May 2015 to April 2020. The patients were divided into two groups: blastocyst transfer group and cleavage-stage transfer group. Among the participants, blastocyst transfer was performed in 30 women and cleavage-stage ET on 209, respectively. Inclusion criteria were: age $\leq 40$ years and $\mathrm{AMH}$ level $<1 \mathrm{ng} / \mathrm{ml}$. Exclusion criteria were: patient reluctance to participate in the study, systemic diseases, women over 40 , and couples with severe male infertility.

\section{Statistical Analysis}

The results of the Klomogrov test show that not all quantitative variables have a normal distribution. MannWhitney test is used to examine the relationship between quantitative variables in the two groups. The Chi-square test and Fisher's exact test are used to examine qualitative variables. For all tests, a significance level of 0.05 will be considered. Data were analyzed using SPSS for Windows, Version 23.0 (Armonk, NY: IBM Corp).

\section{RESULTS}

Among 239 participants, 30 (12.6\%) were in the blastocyst group, and 209 (87.4\%) were in the cleavage group. The participants' age was a minimum of 20 and a maximum of 40 , with a mean of $33.6( \pm 4.9)$. Participants' body mass index (BMI) was a minimum of 19 and a maximum of 29 , with a mean of $24.2( \pm 2.10)$. The number of ART attempts averaged $1.6( \pm 1.60)$ with a minimum of 0 and a maximum of 9 times. The mean AMH level in participants was $0.47( \pm 0.18)$ with a minimum of 0.1 and a maximum of 0.9 . Among the participants, the minimum total number of oocytes was 1 , and the maximum was 13 , with a mean of $3.4( \pm 1.8)$. The mean number of mature oocytes (MII) was $2.7( \pm 1.5)$, with a minimum of 1 and a maximum of 11. Out of 239 participants, 219 (91.6\%) did not smoke, and $20(8.4 \%)$ smoked. Table 1 shows these characteristics. All patients were primary inferility.

\begin{tabular}{|c|c|c|c|c|c|}
\hline Variable & $\mathbf{N}$ & Minimum & Maximum & Mean & $\begin{array}{c}\text { Standard } \\
\text { deviation } \\
\text { (SD) }\end{array}$ \\
\hline Age & 239 & 20 & 40 & 33.6 & 4.9 \\
\hline BMI & 239 & 19 & 29 & 24.2 & 2.10 \\
\hline $\begin{array}{l}\text { Number of } \\
\text { attempts }\end{array}$ & 239 & 0 & 9 & 1.6 & 1.60 \\
\hline $\mathrm{AMH}$ & 239 & 0.1 & 0.9 & 0.47 & 0.18 \\
\hline Total oocyte & 239 & 1 & 13 & 3.4 & 1.8 \\
\hline $\begin{array}{l}\text { Number } \\
\text { of mature } \\
\text { oocytes (MII) }\end{array}$ & 239 & 1 & 11 & 2.7 & 1.5 \\
\hline Smoke & & \multicolumn{2}{|c|}{ Number } & \multicolumn{2}{|c|}{ Frequency } \\
\hline No & & \multicolumn{2}{|c|}{219} & \multicolumn{2}{|c|}{91.6} \\
\hline Yes & & \multicolumn{2}{|c|}{20} & \multicolumn{2}{|r|}{8.4} \\
\hline \multicolumn{6}{|c|}{ Group } \\
\hline \multicolumn{2}{|c|}{ Blastocyte transfer } & \multicolumn{2}{|c|}{30} & \multicolumn{2}{|r|}{12.6} \\
\hline \multicolumn{2}{|c|}{ Cleavage transfer } & \multicolumn{2}{|c|}{209} & \multicolumn{2}{|r|}{87.4} \\
\hline
\end{tabular}

Out of 239 participants, one embryo was transferred to $188(78.3 \%)$ participants, and two embryos were transferred to $51(21.3 \%)$ participants. Examination of participants' pregnancy results showed that 108 (45.2\%) participants had positive pregnancy results, and 131 (54.8\%) participants had negative pregnancy results. Of the 108 participants who tested positive for pregnancy, $83(76.8 \%)$ had a live birth, and 25 (23.1\%) had a miscarriage. These results are shown in Table 2.

\begin{tabular}{|lcc|}
\hline \multicolumn{3}{|l|}{ Table 2. Descriptive statistics of pregnancy results } \\
\hline Pregnancy result & Number & Frequency (\%) \\
\hline Live birth & 83 & 34.7 \\
Missed & 25 & 10.5 \\
Negative & 131 & 54.8 \\
Result & & \\
$\quad$ Positive & 108 & 45.2 \\
$\quad$ Negative & 131 & 54.8 \\
Number of embryos transferred & & \\
1 & 188 & 78.7 \\
2 & 51 & 21.3 \\
\hline
\end{tabular}

Mann-Whitney test was used to evaluate the quantitative variables between the two groups of blastocyst transfer and cleavage-stage transfer. There was no significant difference between the age $(\mathrm{p}=0.2)$ and BMI $(\mathrm{p}=0.4)$ of the two groups. There was also no significant difference between $\mathrm{AMH}(\mathrm{p}=0.3)$ and the number of attempts of the two groups $(\mathrm{p}=0.2)$. There was no significant difference between total oocytes between the two groups $(p=0.1)$. The MII variable was significantly different between the two groups $(\mathrm{p}=0.006)$. The results of this test are given in Table 3 . 


\begin{tabular}{|c|c|c|c|}
\hline \multirow[b]{2}{*}{ Variable } & \multicolumn{2}{|c|}{ Group } & \multirow[b]{2}{*}{ p value } \\
\hline & $\begin{array}{l}\text { Blastocyte } \\
\text { transfer } \\
\text { mean }(\mathrm{Sd})\end{array}$ & $\begin{array}{c}\text { Cleavage } \\
\text { transfer } \\
\text { mean (Sd) }\end{array}$ & \\
\hline Age & $32.9(3.9)$ & $33.7(5.04)$ & 0.2 \\
\hline BMI & $24(2.3)$ & $24.3(2.1)$ & 0.4 \\
\hline Number of attempts & $1.3(1.7)$ & $1.7(1.6)$ & 0.2 \\
\hline $\mathrm{AMH}$ & $0.5(0.2)$ & $0.4(0.2)$ & 0.3 \\
\hline Total oocyte & $4.03(2.2)$ & $3.4(1.8)$ & 0.1 \\
\hline $\begin{array}{l}\text { Number of mature } \\
\text { Oocytes (MII) }\end{array}$ & $3.5(1.8)$ & $2.6(1.4)$ & 0.006 \\
\hline
\end{tabular}

Chi-square and Fisher's exact tests were used to evaluate the qualitative variables between the two groups of blastocyst transfer and cleavage transfer. Examining pregnancy results between the two groups showed no significant difference between positive and negative pregnancy tests $(\mathrm{p}=0.4)$. Even though a higher live birth rate for the blastocyte transfer group was observed with 15 (50\%), no significant difference was found between the number of live births and miscarriages in the two groups $(\mathrm{p}=0.1)$. There was a significant difference in the number of transferred embryos between the two groups $(\mathrm{p}<0.001)$ (Table 4).

Table 4. The comparison of pregnancy results between groups

\begin{tabular}{|c|c|c|c|}
\hline \multirow[b]{2}{*}{ Variable } & \multicolumn{2}{|c|}{ Group } & \multirow[b]{2}{*}{$p$ value } \\
\hline & $\begin{array}{c}\text { Blastocyte } \\
\text { transfer } \\
\text { frequency }(\%)\end{array}$ & $\begin{array}{c}\text { Cleavage } \\
\text { transfer } \\
\text { frequency }(\%)\end{array}$ & \\
\hline Pregnancy result & & & 0.1 \\
\hline Live birth & $15(50)$ & $68(32.5)$ & \\
\hline Missed & $1(3.3)$ & $24(11.5)$ & \\
\hline Negative & $14(46.7)$ & $117(56)$ & \\
\hline Result & & & 0.4 \\
\hline Positive & $16(53.3)$ & $92(44)$ & \\
\hline Negative & $14(46.7)$ & $117(56)$ & \\
\hline \multicolumn{2}{|c|}{ Number of embryos transferred } & & 0.000 \\
\hline 1.00 & $11(36.7)$ & $177(84.7)$ & \\
\hline 2.00 & $19(63.3)$ & $32(15.3)$ & \\
\hline
\end{tabular}

\section{DISCUSSION}

In the present study, pregnancy outcomes were compared in two groups: the cleavage-stage transfer vs. the blastocyst transfer in patients with low AMH levels. Currently, age, antral follicle count (AFC), and AMH levels are generally acknowledged as the best predictors for ovarian reserve (1). In the last decade, despite the results of contradictory clinical trials, there has been an increasing trend towards blastocyst transfer (2). Numerous studies have shown that the blastocyst transfer method can produce viable, genetically more normal embryos (1-6). Our results showed that no significant difference was found between the two groups despite of a higher live birth rate in the blastocyst transfer group. Miscarriage rates were also not significantly different between the two groups. This result is consistent with the results of previous studies (7-9). Jones et al. (10) showed that continuing to culture embryos until the fifth day could provide more competent embryos. However, they concluded that blastocyst transfer could not completely prevent chromosomal abnormalities. Probability of success with IVF cycle largely depends on a woman's ovarian reserve and her ability to produce a large number of high-quality mature oocytes in a cycle after $\mathrm{COH}$. Average serum AMH is $4 \mathrm{ng} / \mathrm{ml}$ in healthy young women with normal ovarian reserve (11). However, a recent consensus reported in La Marca et al. (12) considered poor response at AMH under $1 \mathrm{ng} / \mathrm{ml}$ and high response when AMH is over $3 \mathrm{ng} / \mathrm{ml}$. Nikmard et al. (13) considered normal AMH range at $1.3-2.6 \mathrm{ng} / \mathrm{ml}$ obtaining good ovarian response and clinical outcomes after ART.Our data showed a pregnancy rate of 53.3\% for the blastocyst transfer group and $44 \%$ for the cleavage group. Despite the higher pregnancy rate in the blastocyst transfer group, this difference is not significant. The results of miscarriage also did not show a significant difference between the two groups. These results are consistent with the results of some studies (14-16). Coskun et al. (17) did not find a difference in pregnancy rate and implantation rates between the blastocyte and cleavage stage transfer. Also, Levron et al. (18) found similar results in their comparing the ET day. Lundqvist et al. (19) showed that increasing embryo culture days did not affect the pregnancy rate and the take-home baby rate.On the other hand, these results are inconsistent with some studies as in Frattarelli (20) and Van der Auwera et al. (21) which showed that the rate of pregnancy and implantation in the blastocyst transfer method is higher than the cleavage transfer method. Wilson et al. (22) and Levron et al. (18) also showed that the blastocyst transfer can increase pregnancy rates in patients over 35 . However, they reported higher miscarriage rates for the blastocyst transfer group in contrast to our study.Schwarzler et al. (23) also found higher take-home baby rates in the blastocyst transfer group than in the cleavage-stage transfer group.The American Society for Reproductive Medicine (ASRM), in its guidelines for blastocyte transfer in ART (24), showed no significant association between increased birth rate per cycle by blastocyst transfer compared to cleavagestage transfer. In our study, no significant differences were observed between blastocyst transfer and cleavage-stage ET group among patients with low AMH levels. All our cases were fresh ET. However, different conditions such as frozen or fresh transfer of the embryo (25), the number of transferred blastocytes (26), or diseases and underlying conditions (27) can affect the results. Therefore, further studies should be performed to further investigate the effect of various factors such as AMH on selecting the best transfer day.In a study conducted at an ART database in Canada, 3206 blastocyst transfers were compared with 
9506 cleavage-stage ET. In contrast to our study the live birth rate was significantly higher following blastocyst transfer than following cleavage-stage transfer (26). Some studies have shown that blastocyst transfer can increase the rate of multiple pregnancies in addition to the pregnancy rate (28-30). Also, Karacan et al. (31) have shown that the rate of multiple pregnancy in blastocyst transfer is significantly higher in blastocyst transfer than in cleavage-stage transfer. In contrast to these studies, we have no multiple pregnancy because of limited number of transferable embryos. In the present study, all participants had $\mathrm{AMH}$ level $<1 \mathrm{ng} / \mathrm{ml}$. Very recently, qualitative embryo characteristics as blastulation and aneuploidy rate in addition to clinical IVF outcomes were analyzed by Morin et al. (32) in a large retrospective study including 3457 patients. The authors reported that women younger than 38 years old with evidence of diminished ovarian reserve (DOR) did not display an oocyte qualitative decline. Indeed, fertilized oocytes retrieved from young patients with DOR formed blastocysts of high quality, euploid and were able to produce live births as those of women of the same age with high AMH values (32). As far as we know, no studies have been performed on the role of AMH in the selection of embryo transfer day. Our results showed no significant difference between the two methods in patients with low levels of AMH. One of the limitations of this study was the lack of a control group for the AMH variable. In future studies, control groups with normal AMH levels should be considered to examine the effects of AMH more accurately in each transfer method. Data from this study were also obtained from a single clinic. In subsequent studies, data from several clinics can be combined to obtain more comprehensive conclusions. Indeed, endometrial receptivity in patients with low $\mathrm{AMH}$ is not affected as it thought, but the only difference is the number of obtained embryos with good quality to select the best ones for ET.

\section{CONCLUSION}

In this study, both blastocyte and cleavage-stage transfer in subjects with low AMH levels were compared. No significant difference was observed between the live birth and miscarriage rate between the two groups. Further studies should be performed considering the control group for $\mathrm{AMH}$ and larger populations to confirm the findings of this study.

\section{ETHICAL DECLARATIONS}

Ethics Committee Approval: Ethical approved this study by Research and Project Development Ethics Committee of Beykoz University (Date: 21.12.2020, Decision No: 2020/04).
Informed Consent: Because the study was designed retrospectively, no written informed consent form was obtained from patients.

Referee Evaluation Process: Externally peer-reviewed.

Conflict of Interest Statement: The authors have no conflicts of interest to declare.

Financial Disclosure: The authors declared that this study has received no financial support.

Author Contributions: All of the authors declare that they have all participated in the design, execution, and analysis of the paper, and that they have approved the final version.

\section{REFERENCES}

1. Lehmann P, Velez MP, Saumet J, et al. Anti-Mullerian hormone (AMH): a reliable biomarker of oocyte quality in IVF. J Assist Reprod Genet 2014; 31: 493-8.

2. Papanikolaou EG, Fatemi H, Venetis C, et al. Monozygotic twinning is not increased after single blastocyst transfer compared with single cleavage-stage embryo transfer. Fertil Steril 2010; 93: 592-7.

3. Källén B, Finnström O, Lindam A, Nilsson E, Nygren K-G, Olausson PO. Blastocyst versus cleavage stage transfer in in vitro fertilization: differences in neonatal outcome? Fertil Steril 2010; 94: 1680-3.

4. Kokkali G, Traeger-Synodinos J, Vrettou C, et al. Blastocyst biopsy versus cleavage stage biopsy and blastocyst transfer for preimplantation genetic diagnosis of $\beta$-thalassaemia: a pilot study. Human Reprod 2007; 22: 1443-9.

5. Mangalraj AM, Muthukumar K, Aleyamma T, Kamath MS, George K. Blastocyst stage transfer vs cleavage stage embryo transfer. J Hum Reprod Sci 2009; 2: 23-6.

6. Milki AA, Jun SH, Hinckley MD, Behr B, Giudice LC, Westphal LM. Incidence of monozygotic twinning with blastocyst transfer compared to cleavage-stage transfer. Fertil Steril 2003; 79: 503-6.

7. Barrenetxea G, de Larruzea AL, Ganzabal T, Jiménez R, Carbonero K, Mandiola M. Blastocyst culture after repeated failure of cleavage-stage embryo transfers: a comparison of day 5 and day 6 transfers. Fertil Steril 2005; 83: 49-53.

8. Chang HJ, Lee JR, Jee BC, Suh CS, Kim SH. Impact of blastocyst transfer on offspring sex ratio and the monozygotic twinning rate: a systematic review and meta-analysis. Fertil Steril 2009; 91: 2381-90.

9. Desai N, Ploskonka S, Goodman LR, Austin C, Goldberg J, Falcone T. Analysis of embryo morphokinetics, multinucleation and cleavage anomalies using continuous time-lapse monitoring in blastocyst transfer cycles. Reprod Biol Endocrinol 2014; 12: $1-10$.

10. Jones GM, Trounson AO, Gardner DK, Kausche A, Lolatgis N, Wood C. Evolution of a culture protocol for successful blastocyst development and pregnancy. Hum Reprod 1998; 13: 169-77.

11. Muttukrishna S, Suharjono H, McGarrigle H, et al. Inhibin B and anti-mullerian hormone: markers of ovarian response in IVF/ ICSI patients?. BJOG 2004; 111: 1248-53.

12. La Marca A, Ferraretti AP, Palermo R, et al. The use of ovarian reserve markers in IVF clinical practice: a national consensus. Gynecol Endocrinol 2016; 32: 1-5.

13. Nikmard F, Aflatoonian B, Hosseini E, et al. A comparative study on the results of agonist and antagonist protocols based on serum AMH levels in patients undergoing intracytoplasmic sperm injection. Int J Reprod Biomed 2016; 14: 769-76. 
14. Glujovsky D, Farquhar C, Quinteiro Retamar AM, Alvarez Sedo $\mathrm{CR}$, Blake D. Cleavage stage versus blastocyst stage embryo transfer in assisted reproductive technology. Cochrane Database Syst Rev 2016; 6: CD002118.

15. Ishihara O, Kuwahara A, Saitoh H. Frozen-thawed blastocyst transfer reduces ectopic pregnancy risk: an analysis of single embryo transfer cycles in Japan. Fertil Steril 2011; 95: 1966-9.

16. Martins W, Nastri C, Rienzi L, Van Der Poel S, Gracia C, Racowsky C. Obstetrical and perinatal outcomes following blastocyst transfer compared to cleavage transfer: a systematic review and meta-analysis. Hum Reprod 2016; 31: 2561-9.

17. Coskun S, Hollanders J, Al-Hassan S, Al-Sufyan H, Al-Mayman $\mathrm{H}$, Jaroudi K. Day 5 versus day 3 embryo transfer: a controlled randomized trial. Hum Reprod 2000; 15: 1947-52.

18. Levron J, Shulman A, Bider D, Seidman D, Levin T, Dor J. A prospective randomized study comparing day 3 with blastocyststage embryo transfer. Fertil Steril 2002; 77: 1300-1.

19. Lundqvist M, Rova K, Simberg N, Lundkvist Ö. Embryo transfer after 2 or 5 days of IVF culture: a retrospective comparison. Acta obstetricia et gynecologica Scand 2002; 81: 126-32.

20. Frattarelli JL, Leondires MP, McKeeby JL, Miller BT, Segars JH. Blastocyst transfer decreases multiple pregnancy rates in in vitro fertilization cycles: a randomized controlled trial. Fertil Steril 2003; 79: 228-30

21. Van der Auwera I, Debrock S, Spiessens C, et al. A prospective randomized study: day 2 versus day 5 embryo transfer. Hum Reprod 2002; 17: 1507-12

22. Wilson M, Hartke K, Kiehl M, Rodgers J, Brabec C, Lyles R Integration of blastocyst transfer for all patients. Fertil Steril 2002; 77: 693-6.

23. Schwärzler P, Zech H, Auer M, et al. Pregnancy outcome after blastocyst transfer as compared to early cleavage stage embryo transfer. Hum Reprod 2004; 19: 2097-102.

24. Blastocyst culture and transfer in clinical-assisted reproduction: a committee opinion. Fertil Steril 2013; 99: 667-72.

25. Zeng M, Su S, Li L. Comparison of pregnancy outcomes after vitrification at the cleavage and blastocyst stage: a meta-analysis. J Assist Reprod and Genet 2018; 35: 127-34

26. Zhu Q, Zhu J, Wang Y, et al. Live birth rate and neonatal outcome following cleavage-stage embryo transfer versus blastocyst transfer using the freeze-all strategy. Reprod Biomed Online 2019; 38: 892-900.

27. Vajta G, Parmegiani L. Cleavage stage vs. blastocyst transfer: a more considerate analysis suggested. Hum Reprod 2020; 35: 2399-400

28. Fragouli E, Alfarawati S, Spath K, Wells D. Morphological and cytogenetic assessment of cleavage and blastocyst stage embryos. Mol Hum Reprod 2014; 20: 117-26.

29. Ishihara O, Araki R, Kuwahara A, Itakura A, Saito H, Adamson GD. Impact of frozen-thawed single-blastocyst transfer on maternal and neonatal outcome: an analysis of 277,042 singleembryo transfer cycles from 2008 to 2010 in Japan. Fertil Steril 2014; 101: 128-33.

30. Maheshwari A, Kalampokas T, Davidson J, Bhattacharya S Obstetric and perinatal outcomes in singleton pregnancies resulting from the transfer of blastocyst-stage versus cleavagestage embryos generated through in vitro fertilization treatment: a systematic review and meta-analysis. Fertil Steril 2013; 100: 1615-21.

31. Karacan M, Ulug M, Arvas A, et al. Comparison of the transfer of equal numbers of blastocysts versus cleavage-stage embryos after repeated failure of in vitro fertilization cycles. J Assist Reprod Genet 2014; 31: 269-74

32. Morin SJ, Patounakis G, Juneau CR, Neal SA, Scott RT, Seli E. Diminished ovarian reserve and poor response to stimulation in patient $<38$ years old: A quantitative but not qualitative reduction in performance Hum Reprod 2018; 33: 1489-98. 